\title{
Avaliação de desgaste por perda de massa de sistema extrator de moldes de injeção de termoplásticos revestidos com filme fino de $D L C$
}

\author{
Diego Chiarello \\ Instituto Federal do Rio Grande do Sul (IFRS) - Campus Caxias do Sul \\ (diego.chiarello@caxias.ifrs.edu.br) \\ Alexandre Luís Gasparin \\ Instituto Federal do Rio Grande do Sul (IFRS) - Campus Caxias do Sul \\ (alexandre.gasparin@caxias.ifrs.edu.br)
}

\begin{abstract}
Resumo: O presente trabalho tem por objetivo, a deposição de filmes finos de DLC sobre componentes do sistema extrator de moldes de injeção, verificando a influência deste revestimento superficial no desgaste desses componentes, sob diferentes configurações de lubrificação superficial. Para esta avaliação, pinos extratores, atualmente produzidos em aço AISI H13 e nitretados à gás, foram revestidos com um filme fino de DLC (carbono tipo diamante, do inglês Diamond Like Carbon) e buchas extratoras foram confeccionadas em AISI P2O e AISI H13, e, posteriormente, temperadas e revenidas. O filme fino de DLC foi depositado em uma câmara com sistema PECVD (deposição química a vapor assistida por plasma, do inglês Plasma Enhanced Chemical Vapor Deposition) auxiliado por confinamento eletrostático de plasma. Um dispositivo que simula o sistema de extração de um molde de injeção foi utilizado para testar o comportamento ao desgaste dos conjuntos pinobucha propostos, em função do aumento do número de ciclos. O desgaste nos pinos foi avaliado quantitativamente por perda de massa, após 100.000 ciclos. Os resultados da perda de massa mostram que para as amostras sem utilização de graxa lubrificante, a taxa de desgaste foi consideravelmente menor. Os pinos que menos apresentaram desgaste foram os dos conjuntos PS e HS, ambos sem utilização de graxa, com perda de 5,400 e 5,467 mg, respectivamente. Com isto, ficou evidenciado a possibilidade de utilização de pinos com revestimento de DLC sem a utilização de graxa lubrificante em sistemas extratores de moldes de injeção e que isto ainda propiciou uma redução considerável na taxa de desgaste.
\end{abstract}

Palavras-chave: Tribologia; DLC; Desgaste; Perda de Massa.

\section{Wear's evaluation by mass loss of extractor system of injection molds of thermoplastics coated with thin film of DLC}

Abstract: This paper has as objective, the deposition of DLC thin films over components of injection mold extractor system, checking the influence of this surface coating in the wear of these components, under different surface lubrication configurations. For this evaluation, extractor pins, currently produced in steel AISI H13, by gas nitriding, was coated with DLC (Diamond Like Carbon) thin films. The DLC thin film has been deposited in a chamber with system PECVD (Plasma Enhanced Chemical Vapor Deposition). A device that simulates of the extraction system of an injection mold was used to test the wear behavior of sets pin-bushing proposed, in function of increased number of cycles. Pin wear was quantitatively assessed by mass loss after 100,000 cycles. The results of mass loss show that for samples without using grease, the wear rate was considerably lower. The pins that showed less wear were PS and HS sets, both without using grease, with a loss of 5.400 and $5.467 \mathrm{mg}$, respectively. Whit this, was evidenced the possibility of using DLC coated pins without use of grease in injection mold extraction systems and that this still provided a considerable reduction in the wear rate.

Keywords: Tribology; DLC; Wear; Mass Loss. 


\section{INTRODUÇÃO}

O desenvolvimento de polímeros com propriedades melhoradas tem chamado a atenção da indústria mundial. Há uma forte tendência, por exemplo, no setor automotivo, onde busca-se a substituição de componentes metálicos por poliméricos, visando ganhos com a diminuição do peso e melhoria da eficiência energética do veículo. $\mathrm{O}$ aumento da produção de peças plásticas, requer constante pesquisa também no que se refere aos moldes de injeção utilizados no processo. (IMIHEZRI et al., 2006)(SHAMEEM et al., 2017)

O processo de moldagem por injeção de termoplásticos, por se tratar de um processo de produção de peças seriadas em larga escala, requer constante evolução quanto aos materiais utilizados em seu ferramental, visando maiores intervalos entre paradas para substituição e lubrificação dos componentes de um molde, buscando assim aumento na eficiência e competitividade. (REIS, 2017) (FU et al., 2019)

Os filmes finos de DLC são um material de carbono amorfo hidrogenado (a$\mathrm{C}: \mathrm{H})$ que tem atraído interesse científico e demonstrado grande sucesso industrial, devido seu excepcional desempenho mecânico e propriedades tribológicas exclusivas, o que abre oportunidade para novas aplicações que podem contribuir solucionando problemas. (CEMIN et al., 2015) (CRESPI et al., 2017)

Visando melhorias quanto aos intervalos entra paradas para manutenção e lubrificação dos moldes de injeção de termoplásticos, este trabalho propõe avaliar a aplicação de filmes finos de DLC sobre pinos extratores de moldes e suas propriedades ao desgaste. Tal estudo propôs quatro diferentes configurações de amostras, com diferentes materiais de bucha extratora e diferentes condições de lubrificação, à fim de verificar qual configuração melhor se adapta à esse sistema tribológico.

\section{MATERIAIS E MÉTODOS}

No tribossistema em estudo, à fim de reproduzir o funcionamento do sistema extrator de um molde de injeção de termoplásticos, foram utilizados pinos e buchas extratores, conforme é utilizado industrialmente. As buchas de extração, foram 
fabricadas em aços AISI P20 e AISI H13; tendo sido usinadas em torno CNC, posteriormente tratadas termicamente por têmpera e revenimento e finalizadas com usinagem interna por eletro erosão à fio, sendo o diâmetro interno de $5 \mathrm{~mm}$ com tolerância de ajuste H7. A têmpera das buchas de AISI H13 foi realizada em forno à vácuo à $1030{ }^{\circ} \mathrm{C}$, com resfriamento à vácuo na pressão de 4 bar seguido de duplo revenimento à $600{ }^{\circ} \mathrm{C}$, por 120 minutos cada; já as buchas de AISI P20, foram temperadas à $860^{\circ} \mathrm{C}$, em forno de atmosfera controlada e resfriamento em óleo, seguido de duplo revenimento à $580^{\circ} \mathrm{C}$, por 120 minutos cada. A dureza média medida na seção transversal das buchas foi de 39,4 HRC para as buchas fabricadas em AISI P20 e de 42,8 HRC nas buchas de AISI H13. A composição química destes materiais foi avaliada por espectrometria de emissão ótica e é mostrada na Tabela 1.

Tabela 1 - Composição química dos aços AISI H13 e AISI P20

Análise Química (em \% w)

\begin{tabular}{l|l|l|l|l|l|l|l|l|l|l}
\hline & $\mathrm{C}$ & $\mathrm{Si}$ & $\mathrm{Mn}$ & $\mathrm{Cr}$ & $\mathrm{Mo}$ & $\mathrm{Ni}$ & $\mathrm{V}$ & $\mathrm{W}$ & Co & Cu \\
\hline $\mathrm{H} 13$ & 0,38 & 1,02 & 0,39 & 4,91 & 1,29 & 0,18 & 0,83 & $<0,1$ & 0,016 & 0,127 \\
\hline P20 & 0,38 & 0,27 & 1,41 & 1,83 & 0,19 & 0,97 & 0,01 & $<0,1$ & 0,102 & 0,126 \\
\hline
\end{tabular}

Os pinos extratores utilizados são do Tipo A, segundo norma DIN 1530A, fabricados em aço AISI H13, nitretados a gás e com acabamento superficial retificado. $\mathrm{Na}$ condição de como fornecido, sua dureza de núcleo é de aproximadamente $40 \mathrm{HRC}$ e dureza superficial de 950-1100 HV. Os pinos possuem diâmetro de $5 \mathrm{~mm}$ com tolerância de ajuste g6 e comprimento de $125 \mathrm{~mm}$. A deposição do filme de DLC, sobre os pinos extratores, foi realizada em um reator PECVD e ocorreu em três etapas. Na primeira, foi realizada uma limpeza com gás hidrogênio (H2). Na sequência, foi depositada uma camada intermediária, através da evaporação de liquido precursor à base de silício hexametildissiloxano (HMDSO) e posteriormente, houve a deposição do filme fino de DLC.

Foram realizados alguns testes, com diferentes parâmetros de ensaio à fim de verificar quais são os parâmetros ideais para revestimento do pino extrator em estudo. Alguns defeitos no filme fino foram verificados, como falta de recobrimento e delaminação do filme por falta de adesão. A figura 1, mostra os principais defeitos observados com a deposição do filme fino de DLC. 
Figura 1 - Defeitos na deposição do filme de DLC. a) falta de recobrimento,

b) delaminação por falta de adesão.

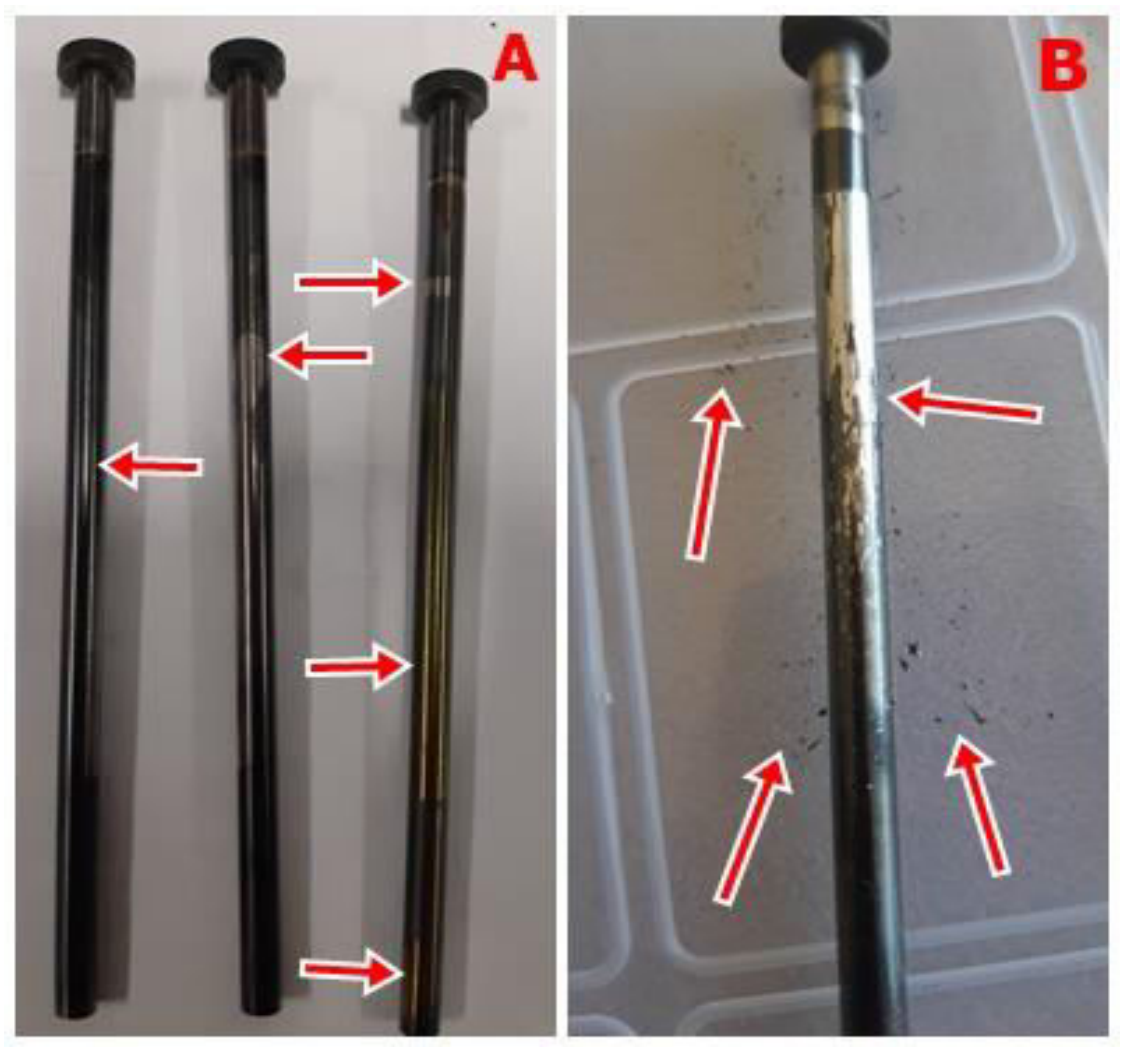

Fonte: autores, 2019.

Em função dos resultados dos testes com diferentes parâmetros, e com base em estudos prévios, foram definidos os parâmetros que seriam utilizados na deposição do DLC nos pinos extratores do estudo. A Tabela 2 mostra os parâmetros selecionados e utilizados no processo de deposição.

Tabela 2 - Parâmetros de processo para limpeza de amostras, deposição de intercamada e deposição de filme de DLC.

\begin{tabular}{llll}
\hline Parâmetros & Limpeza & Intercamada & DLC \\
\hline Pressão (Pa) & 60 & 53 & 53 \\
Precursor & $\mathrm{H}_{2}$ & $\mathrm{Ar}+\mathrm{HMDSO}$ & $\mathrm{Ar}+\mathrm{C}_{2} \mathrm{H}_{2}$ \\
Taxa de Fluxo & $10 \mathrm{sccm}$ & $7 \mathrm{sccm} / 10 \mathrm{~g} / \mathrm{L}$ & $7 / 10 \mathrm{sccm}$ \\
Tensão (V) & -400 & -500 & -800 \\
Tempo (min) & 6 & 10 & 60 \\
Temperatura & & & 300 \\
$\left({ }^{\circ} \mathbf{C}\right)$ & 300 & 300 & \\
\hline
\end{tabular}

Fonte: autores, 2019.

Para a avaliação de desgaste dos pares pino/bucha, utilizou-se um dispositivo que simula o sistema de extração de um molde de injeção de termoplásticos. O 
dispositivo é composto basicamente por uma placa fixa, onde são posicionadas as buchas temperadas as quais simulam os furos de extração do macho de um molde de injeção, e por uma placa móvel guiada por sistema de rolamento linear, onde são fixados os pinos extratores, revestidos com DLC. O movimento é realizado por um sistema pneumático constituído de um cilindro de dupla ação e uma válvula duplo solenoide. O comando elétrico do dispositivo possui um sistema digital para a contagem de ciclos. A usinagem dos componentes do dispositivo foi realizada seguindo as mesmas tolerâncias de ajuste utilizadas para fabricação de moldes de injeção, para simular os ajustes dos moldes de injeção. A figura 2 apresenta o dispositivo utilizado. (REIS, 2017)

Figura 2. Dispositivo utilizado para ensaio de desgaste pino/bucha. a) quadro de comando, b) válvula pneumática, c) cilindro pneumático, d) placa móvel, e) placa fixa, f) base de fixação do sistema, g) sistema de guia linear.

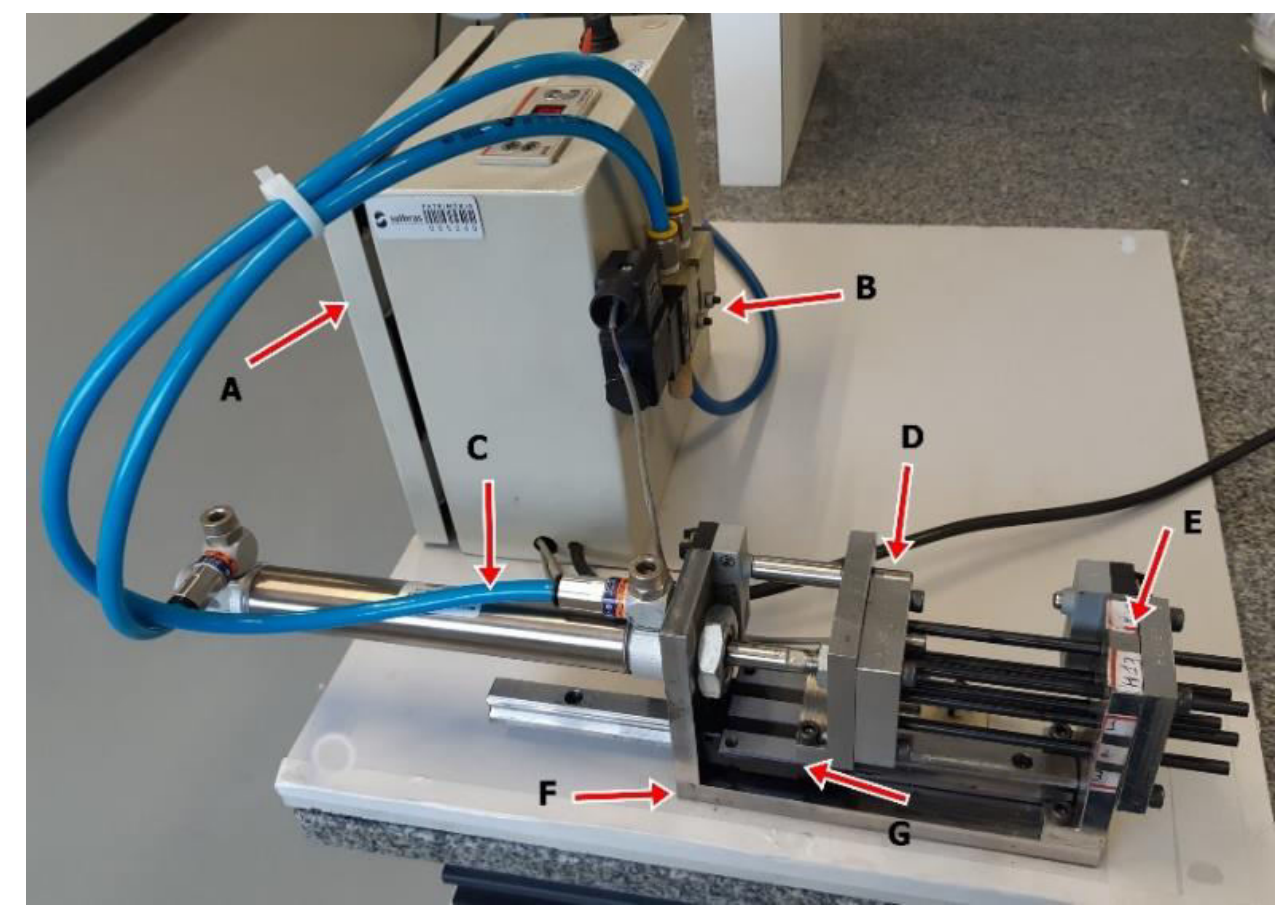

Fonte: autores, 2019.

O sistema de movimentação do dispositivo de ensaio possui um curso linear de $50 \mathrm{~mm}$, o qual totaliza uma distância percorrida de $100 \mathrm{~mm}$ por ciclo. Placa móvel e placa fixa possuem capacidade de montagem de 6 pinos e 6 buchas simultaneamente. A velocidade utilizada no ensaio foi de 0,19 m/s. Para quantificar a perda de massa ocorrida nos pinos e buchas em estudo, foi estipulado a quantidade de 100.000 ciclos, valor este, utilizado industrialmente entre paradas para lubrificação dos componentes de moldes de injeção. A massa dos pinos e buchas foi 
medida, antes e depois da aplicação destes ao ensaio de desgaste, em uma balança analítica, com capacidade de $220 \mathrm{~g}$ e resolução de $0,1 \mathrm{mg}$.

Foram propostas 4 diferentes configurações de amostras; sendo utilizadas buchas de 2 materiais distintos (AISI P20 e AISI H13). Para cada um desses materiais, foram testadas 2 configurações de lubrificação (com lubrificação e sem lubrificação), com o intuito de verificar o comportamento ao desgaste do filme fino de DLC, sob diferentes condições de lubrificação, quando exposto ao deslizamento linear cíclico. Todas as 4 configurações foram testadas com pinos extratores revestidos com DLC, depositados sob as mesmas condições e parâmetros. Todos os pinos e buchas utilizados foram numerados para garantir que as medições quantitativas após o ensaio de desgaste sejam corretas. Os conjuntos de amostras pino/bucha, bem como a denominação dada a cada um deles, estão mostrados na Tabela 3.

Tabela 3 - Configuração de conjuntos de amostras pino/bucha para ensaio de desgaste

\begin{tabular}{llll}
\hline $\begin{array}{l}\text { Denominação } \\
\text { conjunto de amostras }\end{array}$ & Pino & Bucha & Lubrificação \\
\hline HC & DLC & H13 & Com \\
HS & DLC & H13 & Sem \\
PC & DLC & P20 & Com \\
PS & DLC & P20 & Sem \\
\hline
\end{tabular}

Fonte: autores, 2019.

Para cada configuração de conjunto de amostras pino/bucha foram utilizados três pinos e três buchas para melhor confiança dos resultados obtidos, totalizando 12 pares de amostras (4 configurações de amostras x 3 amostras). Para os pares pino/bucha que foi designado o uso de lubrificação, foi utilizada graxa branca para moldes à base de sabão de lítio IGLS-B2, marca Implastec, de grau NGLI 2, aplicada sobre os pinos antes do início dos testes de desgaste.

\section{RESULTADOS E DISCUSSÕES}

A figura 3 mostra os conjuntos pino/bucha antes do ensaio de desgaste. Notase pela uniformidade na coloração superficial, que houve completo recobrimento, 0 que indica que os defeitos observados anteriormente não se repetiram e que a escolha dos parâmetros ideais para de posição do filme de DLC foi eficaz. 
Figura 3 - Conjuntos Pino/Bucha antes do ensaio de desgaste.

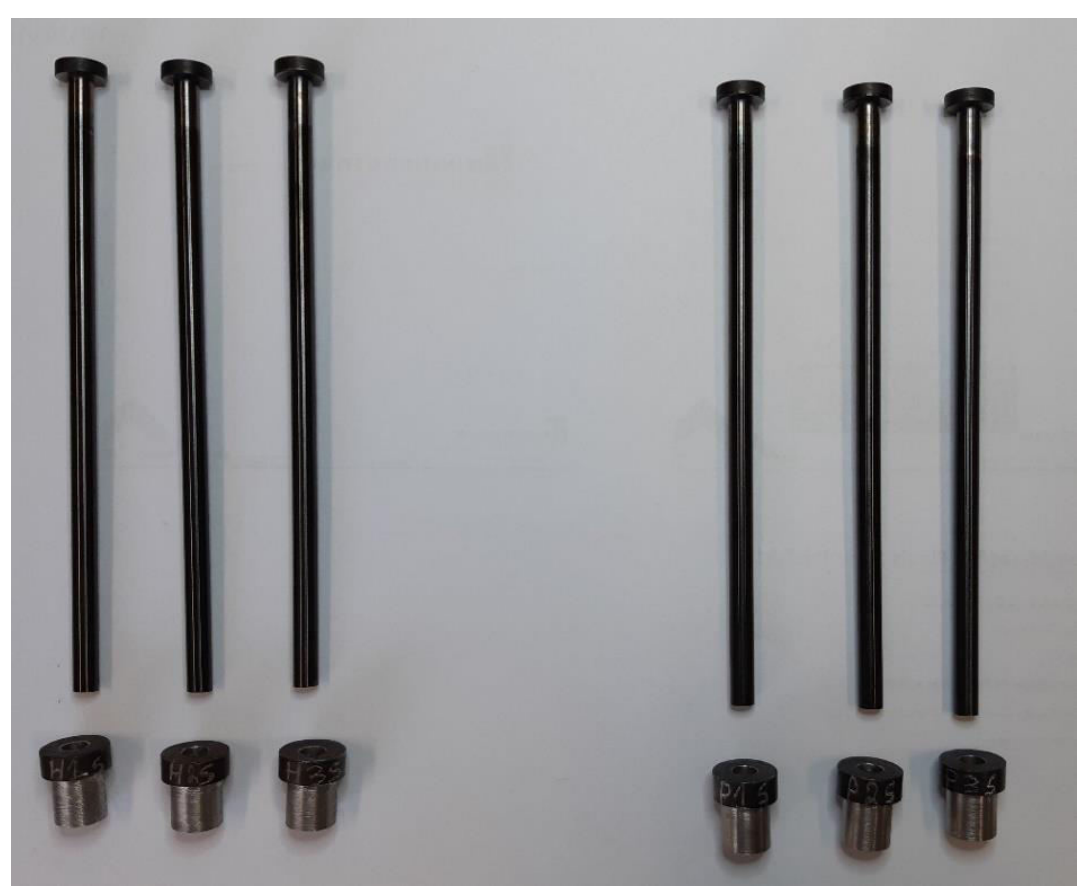

Fonte: autores, 2019.

Após a ciclagem até o número de 100.000 ciclos, o sistema foi parado e as buchas e pinos retirados do equipamento para limpeza com algodão e acetona (Propanona) P.A., para retirada de resíduos de graxa ou fragmentos dos componentes que se desprenderam durante o ensaio.

A figura 4 apresenta os resultados de perda de massa, em mg, para cada um dos componentes e também do conjunto pino/bucha. Os valores representados são a média de três amostras que foram utilizadas para cada uma das quatro configurações de amostragem diferentes.

Dos quatro conjuntos propostos, sendo: bucha AISI H13 com lubrificação, bucha AISI H13 sem lubrificação, bucha AISI P20 com lubrificação e bucha AISI P20 sem lubrificação, os pinos que sofreram menos desgaste foram os dos conjuntos denominados HS e PS, ambos sem a utilização de graxa, com perda de 5,467 e 5,4 mg respectivamente; já as buchas que menos sofreram perda de massa foram as dos conjuntos HC e PC, ambas com utilização de graxa, com diminuição de 2,133mg cada. 
Figura 4 - Perda de massa dos pinos e buchas no ensaio de desgaste sob diferentes condições de lubrificação.

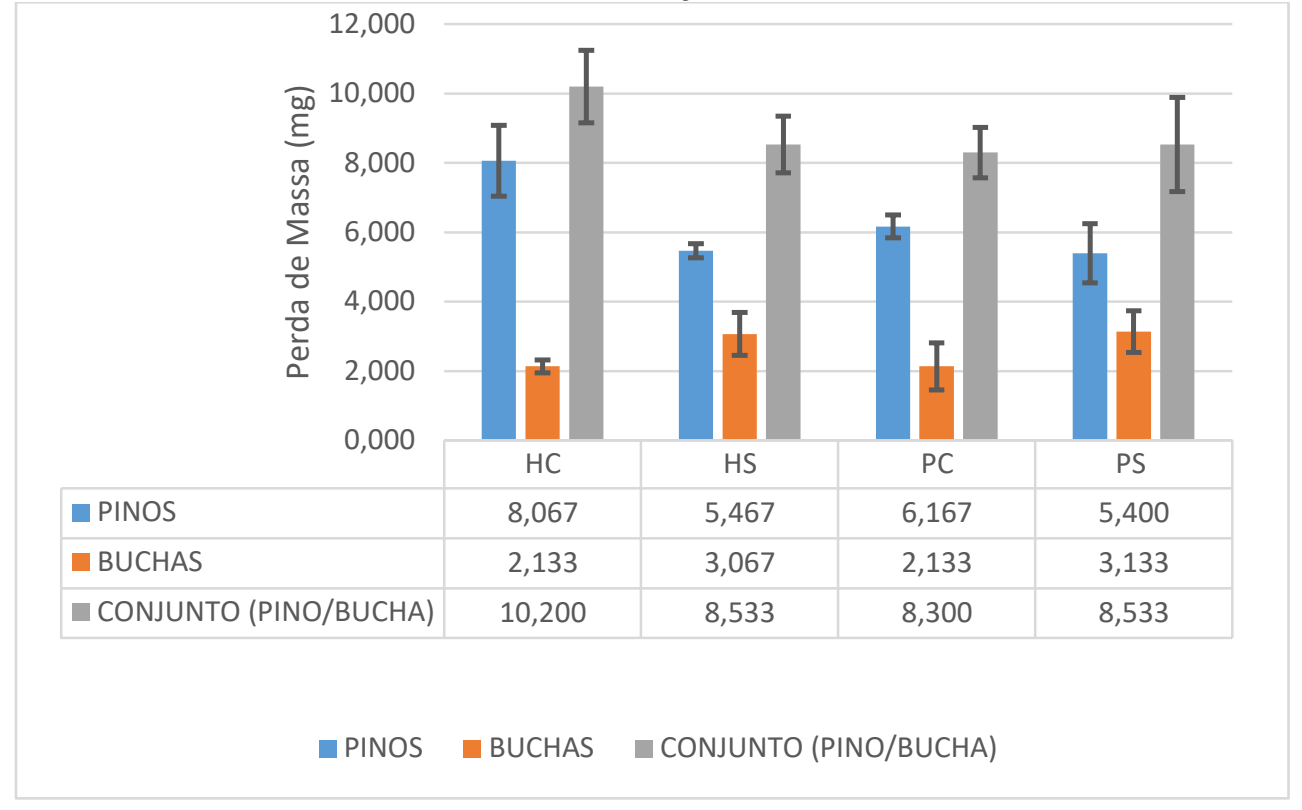

Fonte: autores, 2019.

No comparativo dos resultados das amostras com e sem lubrificação, constata-se que para tanto os conjuntos com buchas AISI H13 quanto com buchas AISI P20, o desgaste dos pinos foi menor quando não ouve a utilização da graxa lubrificante. No caso dos conjuntos com material AISI P20, o desgaste dos pinos foi $12,4 \%$ menor quando não se utilizou a graxa, já nos conjuntos com material AISI H13, quando não se utilizou graxa, o desgaste nos pinos foi $32,2 \%$ menor. Estes indícios apontam que nas amostras em que se utilizou graxa, esta possa ter atuado como um agente abrasivo entre a bucha extratora e o pino revestido com DLC promovendo o arranchamento de micropartículas do filme fino, fato este que atenuou o desgaste do filme de DLC.

Tal fato, comprova que é possível utilizar pinos extratores com revestimento de filmes finos de DLC sem comprometer o processo de injeção e ainda, obter ganhos com a diminuição no desgaste dos pinos extratores, propiciando um aumento nos intervalos entre paradas para manutenção deste e dos demais componentes do molde.

Quando se comprova a possibilidade de operação do sistema extrator dos moldes sem a utilização de graxa lubrificante, obtém-se outra grande vantagem no que se refere à contaminação das peças injetadas. A graxa utilizada como lubrificante dos componentes mecânicos de um molde de injeção, pode entrar em 
contato com o material no interior da cavidade do molde e contaminar a peça injetada. Tal contaminação, dependendo da aplicação da peça, acarreta na necessidade de adição de uma etapa de limpeza ou ainda acaba por impossibilitar a utilização desta peça pois a contaminação por graxa impede que a peça possa receber acabamento superficial de pintura ou metalização, por exemplo. (BLASIO, 2007; RANZAN, 2018)

\section{CONSIDERAÇÕES FINAIS}

A aplicação de revestimento superficial em pinos extratores, com a finalidade de melhoria de propriedades ao desgaste, se mostrou eficaz. Os parâmetros escolhidos para deposição do filme fino de DLC em câmara com sistema PECVD mostraram-se satisfatórios, pois o revestimento cobriu toda a superfície das amostras, apresentando boa adesão à superfície. Os resultados tem grande relevância pois os parâmetros utilizados são de um caso prático, o que remete à um nível alto de confiança caso os materiais e parâmetros venham a ser utilizados industrialmente, na produção de peças injetadas.

Foi possível também, verificar a influência da utilização de diferentes materiais nas buchas extratores e de diferentes condições de lubrificação. Houve uma grande diminuição na taxa de desgaste quando não se utilizou graxa, apesar do grande ganho que isto representa, são necessárias outras caracterizações para explicar com clareza o motivo deste comportamento.

Como perspectivas futuras, pretende-se realizar caracterizações das amostras à fim de entender melhor o comportamento observado nos resultados. Através da realização de Microscopia Eletrônica de Varredura será possível determinar a morfologia e estrutura do filme depositado e entender os mecanismos de desgaste atuantes no par tribológico pino/bucha, além de ajudar a esclarecer porque o uso de graxa resultou em maior desgaste do revestimento de DLC dos pinos extratores. Com a realização de teste de riscamento (Scratch Test), será possível medir o coeficiente de atrito do DLC e mensurar a força de adesão que o filme fino possui com o material do substrato, o que possibilitará explicar melhor o comportamento do desgaste deste material. 


\section{REFERÊNCIAS}

\section{BLASIO, C. A. DE. Solução de Defeitos na Moldagem por Injeção de}

Termoplásticos. [s.I.] Universidade Estadual de Campinas, 2007.

CEMIN, F. et al. The influence of different silicon adhesion interlayers on the tribological behavior of DLC thin films deposited on steel by EC-PECVD. Surface and Coatings Technology, v. 283, p. 115-121, 2015.

CRESPI, A. E. et al. Influence of hydrogen etching on the adhesion of coated ferrous alloy by hydrogenated amorphous carbon deposited at low temperature. Vacuum, 2017.

FU, X. et al. A three-level particle swarm optimization with variable neighbourhood search algorithm for the production scheduling problem with mould maintenance. Swarm and Evolutionary Computation, p. 100572, 2019.

IMIHEZRI, S. S. S. et al. Mould flow and component design analysis of polymeric based composite automotive clutch pedals. Journal of Materials Processing Technology, v. 171, n. 3, p. 358-365, 2006.

RANZAN, T. Reciclagem por Injeção e nova Cromagem de Peças em ABS Cromadas Refugadas. [s.I.] Universidade Regional de Joinville - Univille, 2018.

REIS, T. M. DOS. Avaliação Tribológica do Tratamento de Nitretação a Plasma com Pós-Oxidação Aplicada em Sistema de Extração de Moldes para Injeção de Termoplásticos. [s.I.] Instituto Federal de Educação Ciência e Tecnologia do Rio Grande do Sul, 2017.

SHAMEEM, K. M. M. et al. A hybrid LIBS-Raman system combined with chemometrics: an efficient tool for plastic identification and sorting. Analytical and Bioanalytical Chemistry, v. 409, n. 13, p. 3299-3308, 2017. 